\title{
Mind-Body Exercises for Nurses with Chronic Low Back Pain: An Evidence-Based Review
}

\author{
Pinky Budhrani-Shani, ${ }^{1,2}$ Donna L. Berry, ${ }^{2}$ Patricia Arcari, ${ }^{3}$ \\ Helene Langevin, ${ }^{4}$ and Peter M. Wayne ${ }^{4}$ \\ ${ }^{1}$ Texas Woman's University, Nelda C. Stark College of Nursing, Houston, TX 77030, USA \\ ${ }^{2}$ Phyllis F. Cantor Center for Research in Nursing \& Patient Care Services, Dana-Farber/Harvard Cancer Center, \\ Boston, MA 02215, USA \\ ${ }^{3}$ Dana-Farber Cancer Institute, Boston, MA 02215, USA \\ ${ }^{4}$ Osher Center for Integrative Medicine, Brigham and Women's Hospital, Harvard Medical School, Boston, MA 02115, USA
}

Correspondence should be addressed to Pinky Budhrani-Shani; pbudhrani@twu.edu

Received 28 February 2016; Accepted 5 June 2016

Academic Editor: Maria H. F. Grypdonck

Copyright ( 2016 Pinky Budhrani-Shani et al. This is an open access article distributed under the Creative Commons Attribution License, which permits unrestricted use, distribution, and reproduction in any medium, provided the original work is properly cited.

Background. Chronic low back pain (CLBP) among nurses is a growing health concern. The multimodal nature of mind-body exercises has potential to impact physiological and psychological processes associated with chronic pain, affording possible advantages over conventional unimodal therapies. This paper summarizes the prevalence of and risk factors for CLBP among nurses, reviews the effectiveness in treating pain and disability of mind-body exercises (yoga and tai chi) for CLBP among the general and nursing population, and describes implications. Methods. Articles, published during or prior to 2015, were systematically identified through the PubMed/MEDLINE, Web of Science, and ScienceDirect databases using the following search terms: nurses, mind-body, integrative, biopsychosocial, yoga, tai chi, back pain, and/or risk factors. Results. Prevalence estimates of CLBP among nurses ranged from $50 \%$ to $80 \%$. Associated risk factors for CLBP included lifestyle and physical, psychological, psychosocial, and occupational factors. No published studies were identified that evaluated yoga or tai chi for nurses with CLBP. Studies in the general population suggested that these interventions are effective in reducing pain and disability and may improve factors/processes predictive of CLBP. Conclusion. This review suggests that evaluating the impact of multimodal interventions such as yoga and tai chi for nurses with CLBP warrants investigation.

\section{Introduction}

Nursing is the fifth largest occupation in the United States (U.S.) and ranks as the largest of the healthcare professions [1]. Due to strenuous workloads and irregular shift schedules, musculoskeletal disorders commonly affect nurses [2-4]. The most common musculoskeletal disorder in nurses is chronic low back pain (CLBP), and this is a highly significant and growing health concern [5]. Nurses' experiences of low back pain can affect their health as well as the quality of care they provide [6]. CLBP among nurses is associated with high direct and indirect costs related to lost workdays and increased compensation claims, and CLBP often becomes a chronic health concern among nurses [7]. The nursing workforce represents an important occupational population within which to identify potential risk factors that contribute to CLBP, to identify key interventions that promote quality of life and work productivity, and to ultimately decrease the financial burden of CLBP [8].

As reviewed by Bernal and colleagues [9] using a "nonstructural" psychological and behavioral model of CLBP, even in cases where an initial injury in an anatomical structure can be identified, the pain experience of an individual will be determined by factors including previous pain experiences, beliefs and fears about low back pain, general and psychosocial health, job dissatisfaction, economic status, education, ongoing litigation, compensation claims, and social wellbeing. This perspective supports a shift in the focus for 
treating CLBP from a unimodal approach to a multimodal approach to intervention, integrating pain and disability management with physical, psychosocial, and behavioral strategies [10].

Studies of treatment options, including combinations of anti-inflammatory or other analgesic medication, muscle relaxants, directed exercise, physical therapy, education, and counseling, and treatment protocols targeting subtypes, have failed to identify an optimal treatment strategy for low back pain [11]. Not surprisingly, both patients and physicians have expressed dissatisfaction with currently available conventional treatment options and a significant number report utilizing complementary and integrative options [12]. Nonpharmacologic treatments recommended for persistent back pain include mind-body components, traditional cognitive behavioral therapy (CBT) [13], and mindfulness-based forms of CBT $[14,15]$. CBT is widely recommended for patients with CLBP; however, patient access to CBT is limited $[16,17]$.

Additional complementary and integrative exercises such as yoga, tai chi, and qigong integrate musculoskeletal training (e.g., strength, flexibility, and coordination), breath training, and a variety of cognitive skills (e.g., body awareness, focused mental attention, and relaxation) [18]. It has been hypothesized that the multimodal nature of complementary and integrative exercises has the unique potential to target and impact multiple physiological and psychological processes associated with chronic pain conditions, thus proving to be more beneficial when compared to conventional unimodal therapies [19]. Results from the National Health Interview Survey documented that among 8196 adults who reported experiencing low back pain in the past three months, mind-body exercises were among the most commonly used complementary therapies (13\%) and were rated as "very helpful" by $43 \%$ [20]. Evidence-based clinical guidelines jointly published by the American College of Physicians and the American Pain Society identified mind-body exercises as treatments for CLBP with moderate evidence of effectiveness [21].

In this review, we first summarize the evidence as it relates to the prevalence, burden, and risk factors for the development of CLBP among nurses. We then introduced the logic, rationale, and evidence supporting the use of mind-body exercises for CLBP, focusing on two popular and promising modalities, yoga and tai chi. We review the evidence for these mind-body exercises for back pain in the general population and, when available, research evidence specific to the nursing population. We conclude by discussing current gaps in knowledge and future research needs, as well as the broader implications of introducing nurses to integrative mind-body exercises.

\section{Materials and Methods}

Electronic literature searches were conducted using the PubMed/MEDLINE, Web of Science, and ScienceDirect databases from inception through January 2015. Search terms were low back pain, nurses, risk factors, mind-body, integrative, biopsychosocial, tai chi, and yoga. Searches were limited to the English language. Reference lists of retrieved articles were then examined to identify any additional references relevant for inclusion that were not captured with the online search strategy.

For studies related to prevalence, burden, and risk factors of low back pain among nurses, we included epidemiological studies, surveys, observational studies, and cross-sectional studies with the greatest emphasis placed on articles published within the last ten years. For studies evaluating mindbody exercises for low back pain among nurses, we included randomized trials, interventional studies, and observational studies. Because our systematic search resulted in no relevant studies on the impact of yoga and tai chi for nurses with CLBP, this section of our results utilizes a narrative approach for summarizing the broader literature of yoga and tai chi for low back pain.

\section{Results and Discussion}

3.1. Prevalence, Burden, and Risk Factors. Among healthcare personnel, nurses have the highest rate of CLBP. Among nurses worldwide, the prevalence of low back pain is $40-90 \%$ [22], and in the U.S. 52\% of nurses report CLBP, with lifetime prevalence up to $80 \%[23,24]$. CLBP is associated with the highest rate of any occupation for workers compensation claims, and approximately $38 \%$ of nurses in the U.S. report having occupation-related back pain severe enough to require temporary work leave and $20 \%$ report changing employment due to back pain [24]. According to the 2012 U.S. Bureau of Labor Statistics, nurses with back injuries required a median of 7 days to recuperate [25]. The average compensation cost for work-related back pain was $\$ 10,698$ per case in 2009 [26].

As mentioned, the difficulty in treating CLPB relates to the multifactorial etiology. Modifiable and nonmodifiable risk factors for CLBP among nurses (Figure 1) include demographics, lifestyle factors, occupational factors, physical factors, psychological factors, and psychosocial factors. The high physical demands associated with patient handling, longer work hours, and more demanding schedules are likely the largest contributing factors in the development of low back pain among nurses [26]. Furthermore, the aging of the nursing workforce may also contribute to this problem as the average age of an RN in the U.S. is approximately 47 years [26].

Lifestyle factors such as diet, sleep patterns, obesity, and smoking may also contribute to CLBP. Due to occupational stress, nurses frequently complained of insufficient time to eat well and exercise. Poor eating habits and exercise habits can contribute to weight gain. Results of a meta-analysis of 33 studies indicated that obesity increased the risk of low back pain and had the strongest association with seeking care for CLPB [27]. Results of the Tobacco Use Supplement-Current Population Surveys reported that the smoking rates among RNs (10.73\%) and licensed practical nurses (20.55\%) were higher compared to other healthcare professionals including physicians (2.31\%), dentists (3.01\%), and pharmacists (3.25\%) [28]. Several epidemiological studies have reported an association between smoking and lumbar pain [29]. Nicotine causes vasoconstriction, which reduces the amount of oxygen and nutrients available to muscles, ligaments, and intervertebral discs, increasing chances for injuries and degenerative 


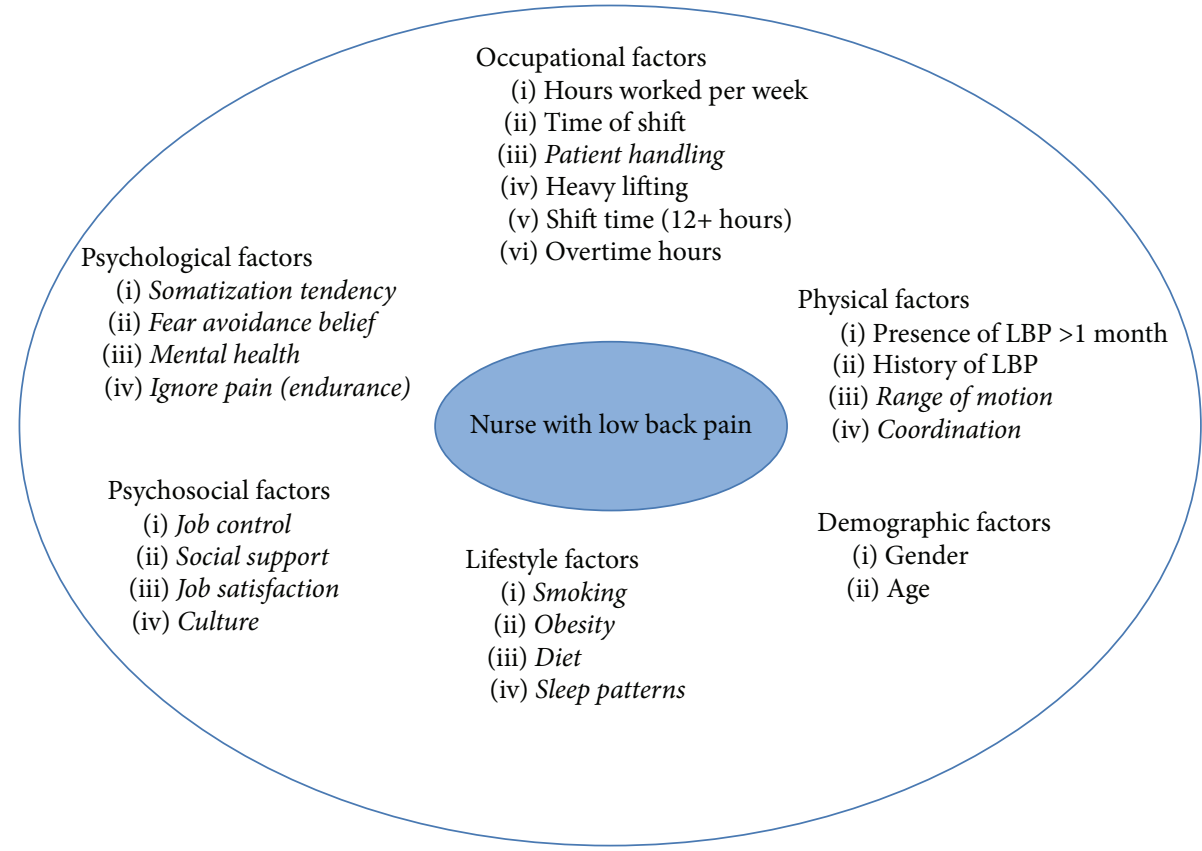

Modifiable risk factors

Nonmodifiable risk factors

FIGURE 1: Proposed model of modifiable and nonmodifiable risk factors associated with chronic low back pain among nurses.

processes in the intervertebral discs to manifest [30]. The biological mechanisms affected by smoking that may be associated with spinal symptoms include coughing reflexes, increased fibrin deposition leading to chronic inflammation, and decreased blood flow and oxygenation of the tissues. These mechanisms affect the metabolic balance of the discs and accelerate degenerative processes leading to increased low back pain and injuries [31].

Occupational related factors for CLBP among nurses include varying shifts, shift time, hours worked per week, patient handling, and heavy lifting. Decreasing RN staff by $9 \%$ led to a $65 \%$ increase in work-related illnesses and injuries among nurses [32]. Furthermore, workday factors including working 13 or more hours per day, varying shifts, working weekends, working with less than 10 hours off between shifts, and working on previously assigned days off were significantly associated with low back pain among nurses [32]. In a systematic review of 89 studies, nursing activities were associated with an increased risk of back disorders regardless of nursing technique, personal characteristics, and non-workrelated factors; patient handling conferred the highest risk of LBP among nurses [33]. Ergonomic factors including bending, carrying patients, torso twisting, and standing increased susceptibility to CLBP [34].

Psychological risk factors for LBP include fear avoidance beliefs, mental health, pain endurance, and somatization tendency $[35,36]$. Results of the international Cultural and Psychosocial Influences in Disability study indicated that established psychological risk factors contribute to LBP among nurses, specifically pessimistic beliefs about pain prognosis, poor mental health, and somatization tendency [37]. In a longitudinal study of 2164 working nurses and midwives with low back pain, fear of movement, passive coping, pain severity, pain radiation, and manual handling frequency were found to significantly increase the likelihood of low back pain [8]. Pain-related fear has been hypothesized to lead to a cycle of decreased movement, connective tissue remodeling, and increased tissue stiffness. Peripheral and central nervous system sensitization may also contribute to tissue inflammation, further decreasing movement, and increasing pain-related fear and distress $[8,38]$.

Psychosocial risk factors for CLBP include culturally influenced health beliefs, social support, job control, and job dissatisfaction. Previous research suggests that interactions of psychosocial factors and physical exhaustion have increased the prevalence of musculoskeletal pain among nurses [39]. One prospective study found that CLBP among nurses' aides was associated with loss of support in workplace and working in night shifts [40]. A survey of 1111 nurses to examine the relationship between individual and work-related psychosocial factors and back pain indicated that low job security was associated with CLBP among nurses [12]. In addition, negative beliefs and low job satisfaction were independently associated with absence from work due to low back pain.

In summary, CLBP among nurses is a prevalent and complex problem. Multiple, interdependent risk factors contribute to the development of CLBP. In their investigative series Injured Nurses, the National Public Radio reported that hospitals can reduce the rate of injuries by investing in lifts, floating mattresses, and full-time coordinators to educate nurses about safe patient handling [41]. In addition, a systematic review including 83 randomized controlled trials 
reported that both exercise therapy and behavioral treatment were effective in reducing pain intensity at short-term followup for individuals with CLBP, compared to a no-treatment control group [42]. We have chosen to focus our review on mind-body exercises for low back pain.

3.2. Mind-Body Exercises for Low Back Pain. Increasingly, exercise is being recommended for the treatment and prevention of CLBP. A 2005 Cochrane Review identified 61 randomized controlled trials (RCTs) (43 of which were specific to CLBP) and concluded that exercise may be effective in decreasing pain and disability and improving function [43]. Interventions to date vary greatly in approach and intended effects (e.g., lumbar stabilization versus lumbar extensor strengthening exercises) $[44,45]$. Reported effects of exercise often have been small, no form has been shown to be consistently better than another, and long-term adherence to exercise programs is often poor [43]. There is some evidence that interventions, which combine exercise with behavioral and/ or educational components, may be more effective than individual therapies [46, 47].

Yoga and tai chi are two increasingly popular mind-body exercises that show promise for the management or treatment of low back pain [48]. Yoga originated in India over 4000 years ago and consists of physical postures ("asanas"), breathing techniques ("pranayama"), and meditation ("dhyana") [49]. Yoga aims to improve physical and emotional balance through the use of postures and breathing techniques [50].

Tai chi (taiji, tai chi chuan, or taijiquan) is a mindbody exercise that originated in China and is growing in popularity in the U.S. $[51,52]$. Tai chi, a practice based on slow intentional movements, often coordinated with breathing and imagery, aims to strengthen and relax the physical body and mind [18]. Tai chi is distinguished from yoga in its greater emphasis on dynamic postural control training and functional applications (tai chi is sometimes referred to as "yoga in motion"). Surveys have suggested that approximately 5 million Americans have practiced tai chi, and this number is increasing [51, 53-55].

\subsection{Impact of Mind-Body Exercise on Chronic Low Back Pain.} The development of CLBP from nonspecific acute pain is complex and impacts a range of physiological and psychosocial processes and symptoms. While virtually no studies are available to evaluate how mind-body exercises impact these processes in nurses with CLBP, a growing body of literature has evaluated mind-body exercises impact on relevant outcomes in other populations. Below, we first summarize the evidence on how mind-body impacts key processes known to underlie CLBP, including risk factors relevant to nurses. We then summarize the clinical outcomes of mind-body exercises for CLBP.

3.4. Impact of Mind-Body Exercise on Risk Factors for Chronic Low Back Pain. There are a number of modifiable risk factors particularly relevant to nurses that can be altered with mindbody exercises. The key processes listed below are particularly relevant to nurses.
3.4.1. Strength and Flexibility. LBP has been associated with weakness in the lower extremities, decreased pelvic strength, and limited lower extremity and torso flexibility [56-58]. Several studies have suggested that yoga can increase muscular strength and joint flexibility among patients with low back pain $[49,59,60]$. Numerous RCTs conducted in diverse populations have demonstrated that tai chi can improve lower extremity strength and increase musculoskeletal flexibility and range of motion in the torso as well as the upper and lower extremities [61-64].

3.4.2. Proprioception and Body Awareness/Sensation. Both yoga and tai chi have been hypothesized to enhance interoceptive, proprioceptive, and kinesthetic awareness [65]. Our literature search did not identify any published studies to date that have evaluated the impact of yoga on these outcomes. Recent cross-sectional and prospective studies suggest that tai chi can improve kinesthetic sense in the ankle, knee, shoulder, and hand [66-68]. Manor and colleagues [69] found that tai chi was associated with improved plantar sensation and physical function in older adults with peripheral neuropathy.

3.4.3. Postural Control. Compared to healthy persons, individuals with LBP have reduced postural control [70-72]. Past studies, including RCTs and observational studies, suggested that yoga improved postural control, mobility, and gait speed among older adults $[73,74]$. A systematic review of 15 studies consisting of 5 RCTs and 4 quasi-experimental, 2 crosssectional, and 4 single-group designs found that yoga may have a beneficial effect on balance [75]. Numerous studies have also reported that tai chi reduces measures of sway (i.e., range of anteroposterior sway, range of mediolateral sway, mean radius, and swept area) as well as multiple outcomes related to balance (e.g., greater single-leg stance time and reduced fear of falling) [64, 76-78]. Other studies suggest that tai chi improves multiple aspects of gait and dynamic postural control [79-82].

3.4.4. Psychological and Behavioral Factors. Individuals with low back pain often have elevated scores on questionnaires measuring pain-related fear of movement and paincatastrophizing cognitive style [83, 84]. Other investigators have reported significant associations between low back pain and depression, anxiety, and somatization [85]. Tekur and colleagues [86] suggested that yoga can reduce pain, anxiety, and depression and improved spinal mobility in patients with low back pain more effectively than physical therapy, suggesting yoga's positive effect on psychological measures. We identified two published studies evaluating eight-week interventions of yoga exercises among nurses with stress [8789]. Results showed that nurses had significantly lower levels of stress and significantly increased confidence in their ability to cope at treatment conclusion (8 weeks) [89] and long-term follow-up (12 months) [88]. Results of a systematic review of 37 randomized controlled studies indicated significant support for the positive effect of tai chi on a variety of psychological symptoms including anxiety, depression, stress, and fear of falling, or improving attitudes such as mood and self-esteem. 
In the included studies, tai chi led to significant improvements in depression compared to routine medication, education controls, waitlist controls, sham exercise controls, martial art controls, and usual daily activity controls [90]. Previous research has also suggested the positive effect of tai chi on psychosocial well-being among individuals with rheumatoid arthritis and fibromyalgia [91, 92]. Twelve weeks of tai chi reduced anxiety and depression and also improved selfefficacy, self-esteem, and motivation among individuals with rheumatoid arthritis [91]. An observational study of 28week tai chi intervention showed improvements in anxiety, depression, self-esteem, and self-efficacy among women with fibromyalgia [92]. We are not aware of any yoga or tai chi studies to date that have specifically evaluated their impact on pain-related fear of movement or pain-catastrophizing cognitive style in patients with low back pain.

3.4.5. Weight Loss. Small studies have reported yoga to be effective for weight loss among obese participants resulting in a decrease in waist circumference, hip circumference, body mass index, and total cholesterol $[93,94]$. Compared to diet education or conventional exercise, limited evidence suggests tai chi may be more effective in reducing body mass index and coronary heart disease risk factors in obese individuals $[95,96]$. We are not aware of any research to date that has been conducted on the impact of tai chi or yoga on weight loss among nurses or in patients with LBP.

\subsection{Clinical Studies on Outcomes of Mind-Body Exercises in the General Population}

3.5.1. Yoga and Back Pain. Our literature search did not identify studies of yoga specifically targeting nurses with CLBP. However, we did identify multiple studies that have evaluated yoga for CLBP in the general population. Three systematic reviews and meta-analyses have summarized this growing body of data. Holtzman and Beggs [97] analyzed efficacy of yoga as an intervention for CLBP in 8 RCTs reporting results from 743 patients. Yoga had a significant positive effect on functional disability and pain. A second meta-analysis included ten RCTs with a total of 967 CLBP patients [98] and provided strong evidence for short-term and long-term positive effects of yoga on pain and back-specific disability. A third systematic review, including 10 clinical trials and a total of 967 participants, also concluded that there is evidence that yoga improves pain and disability in patients with CLBP [99]. While the conclusions of these three reviews generally support the potential of yoga for CLBP, it is important to note that some individual trials included in these reviews primarily reported limited or short-term benefits of yoga for CLBP $[97,98]$.

Two large, methodologically sound yoga trials for CLBP are briefly summarized to characterize the magnitude and scope of reported clinical effects. The first study is a threearm parallel group stratified controlled trial of 228 adults with CLBP randomized to receive 12 weekly classes of yoga compared to conventional stretching exercises or a selfcare book [100]. At the 12-week follow-up, yoga was more effective than the self-care book, but not stretching classes, in improving function and reducing symptoms due to CLBP. The second study was an RCT of 159 employees with low back pain, evaluating the cost-effectiveness of medical yoga compared with evidence-based exercise therapy and self-care advice for nonspecific low back pain. Results suggested that yoga was a cost-effective intervention as noted by differences in production loss due to fewer sickness absence days in the medical yoga group balancing the cost of implementing the medical yoga. From an employer's standpoint, medical yoga costs more than self-care advice but is beneficial due to the improved quality of life and positive incremental health benefits for employees [99]. From a societal perspective, the health gains from medical yoga are cost efficient based on a reduction in sickness absences [100, 101]. Of note, yoga appears to be safe for CLBP as reported by Sherman and colleagues [100] and further supported by conclusions in systematic reviews $[97,98]$.

3.5.2. Tai Chi and Back Pain. As with yoga, we found no studies specifically evaluating tai chi for nurses with CLBP. Published trials of tai chi for back pain in the general population are far fewer in number. In one randomized trial [102] of 160 healthy individuals with nonspecific low back pain, tai chi was compared with a waitlisted control group. Compared to the control group, 10 weeks of tai chi reduced distress associated with back symptoms and pain intensity and improved selfreport disability. Cho [103] reported that 4 weeks of tai chi was effective in reducing acute low back pain when compared to stretching among young Korean males. Two RCTs reported increased flexibility and decreased pain $[104,105]$. There is compelling evidence that tai chi positively impacts multiple musculoskeletal and pain conditions other than CLBP [106-108].

We identified one published study evaluating tai chi among older female nurses with high perceived stress [109]. The design consisted of a small RCT in which 11 nurses with work-related musculoskeletal disorders were randomized to a 15-week tai chi program or a no intervention control group. The tai chi group showed significant improvements in general and mental health, greater reduction in work and general stress, and improvement in trunk flexibility compared to the control group. The tai chi group took no unscheduled time-off hours, had a 3\% increase in work productivity, and had significant improvement in functional reach, whereas the control group had unscheduled absence of 49 hours during the study period. Results suggested that tai chi may be a cost effective wellness option in the workplace. With respect to safety, a systematic review of 153 randomized controlled trials of tai chi concluded that tai chi was not associated with serious adverse events but may be associated with minor musculoskeletal aches and pains [110].

3.6. Limitations and Future Research Directions. There are a number of limitations to this review. One limitation of this study is the broad nature of the question we ask, which intersects with many models and disciplines and thus includes terminology, which is rapidly evolving such as complementary and alternative medicine, integrative medicine, biopsychosocial, and mind-body. While our search was limited to 
key words such as low back pain, nurses, risk factors, integrative, and biopsychosocial, we are confident that significant trials on the key focus of our review, yoga and tai chi for CLBP in nurses, were not missed. Second, our study only included trials published in the English language. Future studies might include other languages for a better global understanding of CLBP among nurses. Finally, our review did not identify any published studies of yoga or tai chi specifically for nurses with CLBP; however, we did find compelling evidence in adult populations with CLBP that yoga and tai chi are safe and likely have positive clinical effects on disability and pain. Other studies in adults support that yoga and tai chi may positively impact multiple physical, behavioral, and psychosocial factors associated with risks of CLBP.

Future studies should be designed with sensitivity to the busy and unusual work schedules of nurses. One possibility would be worksite implementation of yoga and/or tai chi for nurses. The worksite is recognized as an appropriate setting for health promotion and disease prevention as this is where employees spend up to $60 \%$ of their day [111]. Investigators implementing a single arm study reported that a 5-minute mindfulness meditation for pediatric intensive care nurses before each work-shift was feasible and revealed significant decreases in stress from baseline to postintervention and one month following the intervention [112]. Another single arm study found that telephonic sessions of meditation led to improvement in general health, decreased stress, decreased work burnout, and improvement in several other areas among 36 nurses [113]. Results were sustained 4 months after the completion of the intervention. Furthermore, the active involvement of nurses in the planning and implementation of these programs may result in positive outcomes and sustainability of the programs. The sustainability of such programs relies on the appropriate use of data to highlight employee productivity, which may substantiate the value of the financial investment in preventive measures [114]. In a systematic review of 51 studies to determine the relationship between return on investment (ROI) and quality of study methodology in workplace health promotion programs, ROI was calculated as (benefits - costs of program)/costs of program [115]. Across all 51 studies, workplace health promotion programs indicated a $138 \%$ ROI.

Community based pragmatic trials may be a feasible design which would allow participants randomized to mindbody exercises to utilize networks of predefined schools or programs in the community which offer a variety of geographic and timing options. The convenience of pragmatic clinical trials that utilize community based mind-body training networks has been posited to enhance study participant recruitment and retention, as well as poststudy continuation of mind-body practice [116, 117].

Given the high prevalence and the complexity of the CLBP, in addition to research targeting rehabilitation and symptom management, educational initiatives that emphasize prevention should also be considered. A systematic review of work-related psychosocial risk factors and musculoskeletal disorders in nurses reported that most preventive strategies in the workplace are focused on ergonomic (proper weightlifting skills) risk factors; however, prevention should also consider reducing stress and improving the psychosocial work environment [9]. Training in nursing school might include curricula on stress management and the potential of mind-body exercises for the prevention and management of CLBP among nurses. In a survey of 342 nursing personnel, over $90 \%$ reported being interested in additional training in mind-body programs [6]. Greater than $50 \%$ of nurses were specifically interested in additional tai chi or yoga training compared to $26 \%$ and $27 \%$ for diet and exercise training, respectively.

Managing and preventing CLBP in nurses will likely enhance resilience and work engagement but may also have a positive impact on the lives that they touch through work. Advanced practice nurses are usually the first to see patients presenting with new signs of illness in the outpatient setting, and they are at the front lines and best suited to provide patients with preventive health education and recommend interventions [118]. Nurses share important relationships with patients in both inpatient and outpatient settings making them ideal candidates to make referrals for mind-body exercises and to receive training and certification themselves so they can share their knowledge with their patients.

\section{Conclusion}

CLBP among nurses is a prevalent and costly concern. To date, no studies of yoga or tai chi as an intervention for nurses with CLBP were identified. Additional research is needed to establish the efficacy of yoga and tai chi for nurses with CLBP and its potential to be implemented as part of healthcare systems. Given the safety of yoga and tai chi, the cost-effectiveness of delivering these interventions, and the outcomes of most studies suggesting their positive effects on risk factors associated with CLBP, these practices could prove to be highly beneficial for nurses. Exposing nurses to holistic mind-body exercises, both for the prevention and for rehabilitation of CLBP, has the potential to simultaneously address health concerns of both the caregivers and their patients.

\section{Competing Interests}

Peter Wayne is the founder and sole owner of the Tree of Life Tai Chi Center. Peter Wayne's interests were reviewed and managed by the Brigham and Women's Hospital and Partner's HealthCare in accordance with their conflict of interests policies. All other authors declare that there is no conflict of interests regarding the publication of this paper.

\section{Acknowledgments}

This work was supported by the Mittelman Family Fund Integrative Nursing Fellowship for Dr. Pinky BudhraniShani. Dr. Peter M. Wayne was supported by a Grant from the National Complementary and Integrative Health of the National Institutes of Health (R01AT005065). 


\section{References}

[1] Occupational Employment and Wages, http://www.bls.gov/ news.release/ocwage.htm.

[2] B. Heiden, M. Weigl, P. Angerer, and A. Müller, "Association of age and physical job demands with musculoskeletal disorders in nurses," Applied Ergonomics, vol. 44, no. 4, pp. 652-658, 2013.

[3] M. H. Long, V. Johnston, and F. Bogossian, "Work-related upper quadrant musculoskeletal disorders in midwives, nurses and physicians: a systematic review of risk factors and functional consequences," Applied Ergonomics, vol. 43, no. 3, pp. 455-467, 2012.

[4] C. C. Caruso and T. R. Waters, "A review of work schedule issues and musculoskeletal disorders with an emphasis on the healthcare sector," Industrial Health, vol. 46, no. 6, pp. 523-534, 2008.

[5] T. Gropelli and K. Corle, "Assessment of nurses' and therapists' occupational musculoskeletal injuries," Medsurg Nursing, vol. 20, no. 6, pp. 297-304, 2011.

[6] K. Kemper, S. Bulla, D. Krueger, M. J. Ott, J. A. McCool, and P. Gardiner, "Nurses' experiences, expectations, and preferences for mind-body practices to reduce stress," BMC Complementary and Alternative Medicine, vol. 11, article 26, 2011.

[7] R. F. Edlich, C. Woodard, and M. J. Haines, "Disabling back injuries in nursing personnel," Journal of Emergency Nursing, vol. 27, no. 2, pp. 150-155, 2001.

[8] A. P. Dawson, P. J. Schluter, P. W. Hodges, S. Stewart, and C. Turner, "Fear of movement, passive coping, manual handling, and severe or radiating pain increase the likelihood of sick leave due to low back pain," Pain, vol. 152, no. 7, pp. 1517-1524, 2011.

[9] D. Bernal, J. Campos-Serna, A. Tobias, S. Vargas-Prada, F. G. Benavides, and C. Serra, "Work-related psychosocial risk factors and musculoskeletal disorders in hospital nurses and nursing aides: a systematic review and meta-analysis," International Journal of Nursing Studies, vol. 52, no. 2, pp. 635-648, 2015.

[10] R. J. Gatchel, Y. B. Peng, M. L. Peters, P. N. Fuchs, and D. C. Turk, "The biopsychosocial approach to chronic pain: scientific advances and future directions," Psychological Bulletin, vol. 133, no. 4, pp. 581-624, 2007.

[11] S. Haldeman and S. Dagenais, "A supermarket approach to the evidence-informed management of chronic low back pain," The Spine Journal, vol. 8, no. 1, pp. 1-7, 2008.

[12] D. M. Urquhart, H. L. Kelsall, V. C. W. Hoe, F. M. Cicuttini, A. B. Forbes, and M. R. Sim, "Are psychosocial factors associated with low back pain and work absence for low back pain in an occupational cohort?” Clinical Journal of Pain, vol. 29, no. 12, pp. 1015-1020, 2013.

[13] R. Chou, A. Qaseem, V. Snow et al., "Diagnosis and treatment of low back pain: a joint clinical practice guideline from the American College of Physicians and the American Pain Society," Annals of Internal Medicine, vol. 147, no. 7, pp. 478-491, 2007.

[14] D. C. Cherkin, K. J. Sherman, B. H. Balderson et al., "Effect of mindfulness-based stress reduction vs cognitive behavioral therapy or usual care on back pain and functional limitations in adults with chronic low back pain," The Journal of the American Medical Association, vol. 315, no. 12, pp. 1240-1249, 2016.

[15] D. C. Cherkin, K. J. Sherman, B. H. Balderson et al., "Comparison of complementary and alternative medicine with conventional mind-body therapies for chronic back pain: Protocol for the Mind-body Approaches to Pain (MAP) randomized controlled trial," Trials, vol. 15, no. 1, article 211, 2014.
[16] D. M. Ehde, T. M. Dillworth, and J. A. Turner, "Cognitivebehavioral therapy for individuals with chronic pain: efficacy, innovations, and directions for research," The American Psychologist, vol. 69, no. 2, pp. 153-166, 2014.

[17] H. Richmond, A. M. Hall, B. Copsey et al., "The effectiveness of cognitive behavioural treatment for non-specific low back pain: a systematic review and meta-analysis," PLoS ONE, vol. 10, no. 8, Article ID e0134192, 2015.

[18] P. M. Wayne and T. J. Kaptchuk, “Challenges inherent to T'ai Chi research: part I-T'ai Chi as a complex multicomponent intervention," Journal of Alternative and Complementary Medicine, vol. 14, no. 1, pp. 95-102, 2008.

[19] P. Wayne and M. Fuerst, The Harvard Medical School Guide to Tai Chi: 12 Weeks to a Healthy Body, Strong Heart, and Sharp Mind, Harvard Health Publications, Boston, Mass, USA, 2013.

[20] Methods, http://www.cdc.gov/nchs/about/major/nhis/methods .htm.

[21] D. M. Eisenberg, J. E. Buring, A. L. Hrbek et al., "A model of integrative care for low-back pain," Journal of Alternative and Complementary Medicine, vol. 18, no. 4, pp. 354-362, 2012.

[22] S. M. Attar, "Frequency and risk factors of musculoskeletal pain in nurses at a tertiary centre in Jeddah, Saudi Arabia: a cross sectional study," BMC Research Notes, vol. 7, no. 1, article 61, 2014.

[23] R. F. Edlich, K. L. Winters, M. A. Hudson, L. D. Britt, and W. B. Long, "Prevention of disabling back injuries in nurses by the use of mechanical patient lift systems," Journal of Long-Term Effects of Medical Implants, vol. 14, no. 6, pp. 521-533, 2004.

[24] Handle with Care Fact Sheet, http://www.nursingworld.org/ MainMenuCategories/ANAMarketplace/Factsheets-and-Toolkits/FactSheet.html.

[25] Nonfatal Occupational Injuries and Illnesss Requiring Days Away from Work, http://www.bls.gov/news.release/archives/ osh2_11082012.pdf.

[26] Safe patient handling training for schools of nursing, https:// www.cdc.gov/niosh/docs/2009-127/.

[27] R. Shiri, J. Karppinen, P. Leino-Arjas, S. Solovieva, and E. Viikari-Juntura, "The association between obesity and low back pain: a meta-analysis," American Journal of Epidemiology, vol. 171, no. 2, pp. 135-154, 2010.

[28] L. Sarna, S. A. Bialous, K. Sinha, Q. Yang, and M. E. Wewers, "Are health care providers still smoking? Data from the 2003 and 2006/2007 tobacco use supplement-current population surveys," Nicotine and Tobacco Research, vol. 12, no. 11, pp. 1167$1171,2010$.

[29] R. F. C. Moreira, T. O. Sato, F. A. Foltran, L. C. C. B. Silva, and H. J. C. G. Coury, "Prevalence of musculoskeletal symptoms in hospital nurse technicians and licensed practical nurses: associations with demographic factors," Brazilian Journal of Physical Therapy, vol. 18, no. 4, pp. 323-333, 2014.

[30] M. Akmal, A. Kesani, B. Anand, A. Singh, M. Wiseman, and A. Goodship, "Effect of nicotine on spinal disc cells: a cellular mechanism for disc degeneration," Spine, vol. 29, no. 5, pp. 568 575, 2004.

[31] M. S. Goldberg, S. C. Scott, and N. E. Mayo, "A review of the association between cigarette smoking and the development of nonspecific back pain and related outcomes," Spine, vol. 25, no. 8, pp. 995-1014, 2000.

[32] A. M. Trinkoff, R. Le, J. Geiger-Brown, J. Lipscomb, and G. Lang, "Longitudinal relationship of work hours, mandatory overtime, and on-call to musculoskeletal problems in nurses," 
American Journal of Industrial Medicine, vol. 49, no. 11, pp. 964971, 2006.

[33] A. Yassi and K. Lockhart, "Work-relatedness of low back pain in nursing personnel: a systematic review," International Journal of Occupational and Environmental Health, vol. 19, no. 3, pp. 223244, 2013.

[34] P. Pillastrini, R. Bonfiglioli, F. Banchelli et al., "The effect of a multimodal group programme in hospital workers with persistent Low Back Pain: a prospective observational study," Medicina del Lavoro, vol. 104, no. 5, pp. 380-392, 2013.

[35] S. Vargas-Prada, C. Serra, J. M. Martínez et al., "Psychological and culturally-influenced risk factors for the incidence and persistence of low back pain and associated disability in Spanish workers: findings from the CUPID study," Occupational and Environmental Medicine, vol. 70, no. 1, pp. 57-62, 2013.

[36] M. Grotle, N. E. Foster, K. M. Dunn, and P. Croft, "Are prognostic indicators for poor outcome different for acute and chronic low back pain consulters in primary care?" Pain, vol. 151, no. 3, pp. 790-797, 2010.

[37] S. Vargas-Prada, J. M. Martínez, D. Coggon, G. Delclos, F. G. Benavides, and C. Serra, "Health beliefs, low mood, and somatizing tendency: contribution to incidence and persistence of musculoskeletal pain with and without reported disability," Scandinavian Journal of Work, Environment \& Health, vol. 39, no. 6, pp. 589-598, 2013.

[38] H. M. Langevin and K. J. Sherman, "Pathophysiological model for chronic low back pain integrating connective tissue and nervous system mechanisms," Medical Hypotheses, vol. 68, no. 1, pp. 74-80, 2007.

[39] R. Mehrdad, J. T. Dennerlein, M. Haghighat, and O. Aminian, "Association between psychosocial factors and musculoskeletal symptoms among Iranian nurses," American Journal of Industrial Medicine, vol. 53, no. 10, pp. 1032-1039, 2010.

[40] W. Eriksen, "The prevalence of musculoskeletal pain in Norwegian nurses' aides," International Archives of Occupational and Environmental Health, vol. 76, no. 8, pp. 625-630, 2003.

[41] At VA Hospitals, Training and Technology Reduce Nurses' Injuries, http://www.npr.org/2015/02/25/387298633/at-va-hospitals-training-and-technology-reduce-nurses-injuries.

[42] M. van Middelkoop, S. M. Rubinstein, T. Kuijpers et al., "A systematic review on the effectiveness of physical and rehabilitation interventions for chronic non-specific low back pain," European Spine Journal, vol. 20, no. 1, pp. 19-39, 2011.

[43] J. A. Hayden, M. W. van Tulder, A. Malmivaara, and B. W. Koes, "Exercise therapy for treatment of non-specific low back pain," The Cochrane Database of Systematic Reviews, no. 3, Article ID CD000335, 2005.

[44] J. Mayer, V. Mooney, and S. Dagenais, "Evidence-informed management of chronic low back pain with lumbar extensor strengthening exercises," Spine Journal, vol. 8, no. 1, pp. 96-113, 2008.

[45] C. J. Standaert and S. A. Herring, "Expert opinion and controversies in musculoskeletal and sports medicine: core stabilization as a treatment for low back pain," Archives of Physical Medicine and Rehabilitation, vol. 88, no. 12, pp. 1734-1736, 2007.

[46] J. Guzmán, R. Esmail, K. Karjalainen, A. Malmivaara, E. Irvin, and C. Bombardier, "Multidisciplinary bio-psycho-social rehabilitation for chronic low back pain," The Cochrane Database of Systematic Reviews, no. 1, Article ID CD000963, 2002.

[47] A. P. Dawson, S. N. McLennan, S. D. Schiller, G. A. Jull, P. W. Hodges, and S. Stewart, "Interventions to prevent back pain and back injury in nurses: a systematic review," Occupational and Environmental Medicine, vol. 64, no. 10, pp. 642-650, 2007.

[48] "2012 Yoga in America Market Study," http://www.yogajournal .com/article/press-releases/yoga-journal-releases-2012-yoga-inamerica-market-study/.

[49] K. A. Carneiro and J. D. Rittenberg, "The role of exercise and alternative treatments for low back pain," Physical Medicine and Rehabilitation Clinics of North America, vol. 21, no. 4, pp. 777792, 2010.

[50] A. Kogler, Yoga, vol. 38, Jelenia Góra, Jelenia Góra, Poland, 1990.

[51] G. S. Birdee, P. M. Wayne, R. B. Davis, R. S. Phillips, and G. Y. Yeh, "T'ai Chi and Qigong for health: patterns of use in the United States," Journal of Alternative and Complementary Medicine, vol. 15, no. 9, pp. 969-973, 2009.

[52] P. M. Barnes, B. Bloom, and R. L. Nahin, "Complementary and alternative medicine use among adults and children: United States, 2007," National Health Statistics Reports, no. 12, pp. 1-23, 2009.

[53] W. C-Zao, National Expert Meeting on Qi Gong and Tai Chi Consensus Report, University of Illinois Urbana-Champaign, Urbana, Ill, USA, 2005.

[54] P. M. Barnes, B. Bloom, and R. L. Nahin, "Complementary and alternative medicine use among adults and children: United States, 2007," NationaL Health Statistics Reports, vol. 10, no. 12, pp. 1-23, 2009.

[55] R. Lauche, P. M. Wayne, G. Dobos, and H. Cramer, "Prevalence, patterns, and predictors of T'ai Chi and qigong use in the united states: results of a nationally representative survey," The Journal of Alternative and Complementary Medicine, vol. 22, no. 4, pp. 336-342, 2016.

[56] C. J. C. Lamoth, O. G. Meijer, A. Daffertshofer, P. I. J. M. Wuisman, and P. J. Beek, "Effects of chronic low back pain on trunk coordination and back muscle activity during walking: changes in motor control," European Spine Journal, vol. 15, no. 1, pp. $23-$ 40, 2006.

[57] S. F. Nadler, G. A. Malanga, M. DePrince, T. P. Stitik, and J. H. Feinberg, "The relationship between lower extremity injury, low back pain, and hip muscle strength in male and female collegiate athletes," Clinical Journal of Sport Medicine, vol. 10, no. 2, pp. 8997, 2000.

[58] M. S. Puniello, C. A. McGibbon, and D. E. Krebs, "Lifting strategy and stability in strength-impaired elders," Spine, vol. 26, no. 7, pp. 731-737, 2001.

[59] T. Field, "Yoga clinical research review," Complementary Therapies in Clinical Practice, vol. 17, no. 1, pp. 1-8, 2011.

[60] K. P. Roland, J. M. Jakobi, and G. R. Jones, "Does yoga engender fitness in older adults? A critical review," Journal of Aging and Physical Activity, vol. 19, no. 1, pp. 62-79, 2011.

[61] C. Lan, J.-S. Lai, S.-Y. Chen, and M.-K. Wong, "12-month Tai Chi training in the elderly: its effect on health fitness," Medicine and Science in Sports and Exercise, vol. 30, no. 3, pp. 345-351, 1998.

[62] W. Y. Sun, M. Dosch, G. D. Gilmore, W. Pemberton, and T. Scarseth, "Effects of a Tai Chi Chuan program on Hmong American older adults," Educational Gerontology, vol. 22, no. 2, pp. 161-167, 1996.

[63] B. Jacobson and H. Chen, "The effect of Tai Chi Chuan training on balance, kinesthetic sense, and strength," Perceptual and Motor Skills, vol. 84, pp. 27-33, 1997.

[64] G. Wu, F. Zhao, X. Zhou, and L. Wei, "Improvement of isokinetic knee extensor strength and reduction of postural sway in the 
elderly from long-term Tai Chi exercise," Archives of Physical Medicine and Rehabilitation, vol. 83, no. 10, pp. 1364-1369, 2002.

[65] L. Schmalzl, M. A. Crane-Godreau, and P. Payne, "Movementbased embodied contemplative practices: definitions and paradigms," Frontiers in Human Neuroscience, vol. 8, no. 1, article 205, 2014.

[66] C. E. Kerr, J. R. Shaw, R. H. Wasserman et al., "Tactile acuity in experienced Tai Chi practitioners: evidence for use dependent plasticity as an effect of sensory-attentional training," Experimental Brain Research, vol. 188, no. 2, pp. 317-322, 2008.

[67] W. W. N. Tsang and C. W. Y. Hui-Chan, "Effects of exercise on joint sense and balance in elderly men: Tai Chi versus golf," Medicine \& Science in Sports \& Exercise, vol. 36, no. 4, pp. 658667, 2004.

[68] W. W. N. Tsang and C. W. Y. Hui-Chan, "Effects of Tai Chi on joint proprioception and stability limits in elderly subjects," Medicine and Science in Sports and Exercise, vol. 35, no. 12, pp. 1962-1971, 2003.

[69] B. Manor, L. A. Lipsitz, P. M. Wayne, C.-K. Peng, and L. Li, "Complexity-based measures inform Tai Chi's impact on standing postural control in older adults with peripheral neuropathy," BMC Complementary and Alternative Medicine, vol. 13, article 87,2013

[70] S. Luoto, H. Aalto, S. Taimela, H. Hurri, I. Pyykkö, and H. Alaranta, "One-footed and externally disturbed two-footed postural control in patients with chronic low back pain and healthy control subjects: a controlled study with follow-up," Spine, vol. 23, no. 19, pp. 2081-2089, 1998.

[71] A. Hamaoui, M. C. Do, L. Poupard, and S. Bouisset, "Does respiration perturb body balance more in chronic low back pain subjects than in healthy subjects?" Clinical Biomechanics, vol. 17, no. 7, pp. 548-550, 2002.

[72] A. Hamaoui, M. C. Do, and S. Bouisset, "Postural sway increase in low back pain subjects is not related to reduced spine range of motion," Neuroscience Letters, vol. 357, no. 2, pp. 135-138, 2004.

[73] K. K. Zettergren, J. M. Lubeski, and J. M. Viverito, "Effects of a yoga program on postural control, mobility, and gait speed in community-living older adults: a pilot study," Journal of Geriatric Physical Therapy, vol. 34, no. 2, pp. 88-94, 2011.

[74] M. Ni, K. Mooney, L. Richards et al., "Comparative impacts of Tai Chi, balance training, and a specially-designed yoga program on balance in older fallers," Archives of Physical Medicine and Rehabilitation, vol. 95, no. 9, pp. 1620-1628.e30, 2014.

[75] P. E. Jeter, A.-F. Nkodo, S. H. Moonaz, and G. Dagnelie, "A systematic review of yoga for balance in a healthy population," Journal of Alternative and Complementary Medicine, vol. 20, no. 4, pp. 221-232, 2014.

[76] P. M. Wayne, D. E. Krebs, S. L. Wolf et al., "Can Tai Chi improve vestibulopathic postural control?" Archives of Physical Medicine and Rehabilitation, vol. 85, no. 1, pp. 142-152, 2004.

[77] C. Wang, J. P. Collet, and J. Lau, "The effect of Tai Chi on health outcomes in patients with chronic conditions: a systematic review," Archives of Internal Medicine, vol. 164, no. 5, pp. 493$501,2004$.

[78] S. Komagata and R. Newton, "The effectiveness of Tai Chi on improving balance in older adults: an evidence-based review," Journal of Geriatric Physical Therapy, vol. 26, no. 2, pp. 9-16, 2003.

[79] B. Manor, M. Lough, M. M. Gagnon, A. Cupples, P. M. Wayne, and L. A. Lipsitz, "Functional benefits of Tai Chi training in senior housing facilities," Journal of the American Geriatrics Society, vol. 62, no. 8, pp. 1484-1489, 2014.
[80] C.-L. Shen, C. R. James, M.-C. Chyu et al., "Effects of tai chi on gait kinematics, physical function, and pain in elderly with knee osteoarthritis-a pilot study," The American Journal of Chinese Medicine, vol. 36, no. 2, pp. 219-232, 2008.

[81] C. A. McGibbon, D. E. Krebs, S. W. Parker, D. M. Scarborough, P. M. Wayne, and S. L. Wolf, "Tai Chi and vestibular rehabilitation improve vestibulopathic gait via different neuromuscular mechanisms: Preliminary report," BMC Neurology, vol. 5, no. 1, article 3, 2005.

[82] C. J. Hass, R. J. Gregor, D. E. Waddell et al., “The influence of Tai Chi training on the center of pressure trajectory during gait initiation in older adults," Archives of Physical Medicine and Rehabilitation, vol. 85, no. 10, pp. 1593-1598, 2004.

[83] I. E. J. Swinkels-Meewisse, J. Roelofs, R. A. B. Oostendorp, A. L. M. Verbeek, and J. W. S. Vlaeyen, "Acute low back pain: painrelated fear and pain catastrophizing influence physical performance and perceived disability," Pain, vol. 120, no. 1-2, pp. 3643, 2006.

[84] I. E. J. Swinkels-Meewisse, J. Roelofs, E. G. W. Schouten, A. L. M. Verbeek, R. A. B. Oostendorp, and J. W. S. Vlaeyen, "Fear of movement/(re)injury predicting chronic disabling low back pain: a prospective inception cohort study," Spine, vol. 31, no. 6, pp. 658-664, 2006.

[85] T. Pincus, A. K. Burton, S. Vogel, and A. P. Field, "A systematic review of psychological factors as predictors of chronicity/disability in prospective cohorts of low back pain," Spine, vol. 27, no. 5, pp. E109-E120, 2002.

[86] P. Tekur, R. Nagarathna, S. Chametcha, A. Hankey, and H. R. Nagendra, "A comprehensive yoga programs improves pain, anxiety and depression in chronic low back pain patients more than exercise: an RCT," Complementary Therapies in Medicine, vol. 20, no. 3, pp. 107-118, 2012.

[87] C. D. N. Rasmussen, A. Holtermann, O. S. Mortensen, K. Søgaard, and M. B. Jørgensen, "Prevention of low back pain and its consequences among nurses' aides in elderly care: a steppedwedge multi-faceted cluster-randomized controlled trial," BMC Public Health, vol. 13, no. 1, article 1088, 2013.

[88] B. Tarantino, M. Earley, D. Audia, C. D’Adamo, and B. Berman, "Qualitative and quantitative evaluation of a pilot integrative coping and resiliency program for healthcare professionals," Explore, vol. 9, no. 1, pp. 44-47, 2013.

[89] T. Mehrabi, F. Azadi, S. Pahlavanzadeh, and N. Meghdadi, “The effect of yoga on coping strategies among intensive care unit nurses," Iranian Journal of Nursing and Midwifery Research, vol. 17, no. 6, pp. 421-424, 2012.

[90] F. Wang, E.-K. O. Lee, T. Wu et al., "The effects of tai chi on depression, anxiety, and psychological well-being: a systematic review and meta-analysis," International Journal of Behavioral Medicine, vol. 21, no. 4, pp. 605-617, 2014.

[91] J. M. Waite-Jones, C. A. Hale, and H.-Y. Lee, "Psychosocial effects of Tai Chi exercise on people with rheumatoid arthritis," Journal of Clinical Nursing, vol. 22, no. 21-22, pp. 3053-3061, 2013.

[92] A. Romero-Zurita, A. Carbonell-Baeza, V. A. Aparicio, J. R. Ruiz, P. Tercedor, and M. Delgado-Fernández, "Effectiveness of a Tai-Chi training and detraining on functional capacity, symptomatology and psychological outcomes in women with fibromyalgia," Evidence-Based Complementary and Alternative Medicine, vol. 2012, Article ID 614196, 9 pages, 2012.

[93] S. Dhananjai, Sadashiv, S. Tiwari, K. Dutt, and R. Kumar, "Reducing psychological distress and obesity through Yoga 
practice," International Journal of Yoga, vol. 6, no. 1, pp. 66-70, 2013.

[94] S. Telles, S. K. Sharma, A. Yadav, N. Singh, and A. Balkrishna, "A comparative controlled trial comparing the effects of yoga and walking for overweight and obese adults," Medical Science Monitor, vol. 20, pp. 894-904, 2014.

[95] N. Beebe, S. Magnanti, L. Katkowski et al., "Effects of the addition of T'ai Chi to a dietary weight loss program on lipoprotein atherogenicity in obese older women," Journal of Alternative and Complementary Medicine, vol. 19, no. 9, pp. 759-766, 2013.

[96] S.-C. Chen, K.-C. Ueng, S.-H. Lee, K.-T. Sun, and M.-C. Lee, "Effect of T'ai Chi exercise on biochemical profiles and oxidative stress indicators in obese patients with type 2 diabetes," Journal of Alternative and Complementary Medicine, vol. 16, no. 11, pp. 1153-1159, 2010.

[97] S. Holtzman and R. T. Beggs, "Yoga for chronic low back pain: a meta-analysis of randomized controlled trials," Pain Research and Management, vol. 18, no. 5, pp. 267-272, 2013.

[98] H. Cramer, R. Lauche, H. Haller, and G. Dobos, "A systematic review and meta-analysis of yoga for low back pain," The Clinical Journal of Pain, vol. 29, no. 5, pp. 450-460, 2013.

[99] B. T. Saragiotto, T. P. Yamato, and C. Maher, "Yoga for low back pain: PEDro systematic review update," British Journal of Sports Medicine, vol. 49, no. 20, p. 1351, 2015.

[100] K. J. Sherman, D. C. Cherkin, R. D. Wellman et al., "A randomized trial comparing yoga, stretching, and a self-care book for chronic low back pain," Archives of Internal Medicine, vol. 171, no. 22, pp. 2019-2026, 2011.

[101] E. Aboagye, M. L. Karlsson, J. Hagberg, and I. Jensen, "Costeffectiveness of early interventions for non-specific low back pain: a randomized controlled study investigating medical yoga, exercise therapy and self-care advice," Journal of Rehabilitation Medicine, vol. 47, no. 2, pp. 167-173, 2015.

[102] A. M. Hall, C. G. Maher, P. Lam, M. Ferreira, and J. Latimer, "Tai chi exercise for treatment of pain and disability in people with persistent low back pain: a randomized controlled trial," Arthritis Care \& Research, vol. 63, no. 11, pp. 1576-1583, 2011.

[103] Y. Cho, "Effects of tai chi on pain and muscle activity in young males with acute low back pain," Journal of Physical Therapy Science, vol. 26, no. 5, pp. 679-681, 2014.

[104] H. Q. Tian, H. Z. Ning, and D. M. Chen, “Taijiquan as adjuvant treatment ofsenility and middle-age lumbar vertebrae disease," Journal of Hubei University for Nationalities-Medical Education, vol. 21, pp. 24-26, 2004.

[105] C. Y. Chin, C. P. Li, Y. Chuei, M. Chian, and H. Y. Guan, "Prevention of falls in the elderly with taijiquan," Chinese Aging, vol. 28, pp. 2055-2056, 2008.

[106] C. Wang, "Tai Chi improves pain and functional status in adults with rheumatoid arthritis: results of a pilot single-blinded randomized controlled trial," Medicine and Sport Science, vol. 52, pp. 218-229, 2008.

[107] C. Wang, M. D. Iversen, T. McAlindon et al., "Assessing the comparative effectiveness of Tai Chi versus physical therapy for knee osteoarthritis: design and rationale for a randomized trial," BMC Complementary and Alternative Medicine, vol. 14, article 333, 2014.

[108] C. Wang, T. McAlindon, R. A. Fielding et al., "A novel comparative effectiveness study of Tai Chi versus aerobic exercise for fibromyalgia: study protocol for a randomized controlled trial," Trials, vol. 16, no. 1, article 34, 2015.
[109] M. V. Palumbo, G. Wu, H. Shaner-McRae, B. Rambur, and B. McIntosh, "Tai Chi for older nurses: a workplace wellness pilot study," Applied Nursing Research, vol. 25, no. 1, pp. 54-59, 2012.

[110] P. M. Wayne, D. L. Berkowitz, D. E. Litrownik, J. E. Buring, and G. Y. Yeh, "What do we really know about the safety of Tai Chi?: a systematic review of adverse event reports in randomized trials," Archives of Physical Medicine and Rehabilitation, vol. 95, no. 12, pp. 2470-2483, 2014.

[111] M. E. Batt, "Physical activity interventions in the workplace: the rationale and future direction for workplace wellness," British Journal of Sports Medicine, vol. 43, no. 1, pp. 47-48, 2009.

[112] T. Gauthier, R. M. L. Meyer, D. Grefe, and J. I. Gold, "An on-thejob mindfulness-based intervention for pediatric ICU nurses: a pilot," Journal of Pediatric Nursing, vol. 30, no. 2, pp. 402-409, 2015.

[113] D. Bazarko, R. A. Cate, F. Azocar, and M. J. Kreitzer, "The impact of an innovative mindfulness-based stress reduction program on the health and well-being of nurses employed in a corporate setting," Journal of Workplace Behavioral Health, vol. 28, no. 2, pp. 107-133, 2013.

[114] G. Alexander, "Self-care and yoga-academic-practice collaboration for occupational health," Workplace Health \& Safety, vol. 61, no. 12, pp. 510-513, 2013.

[115] S. Baxter, K. Sanderson, A. J. Venn, C. L. Blizzard, and A. J. Palmer, "The relationship between return on investment and quality of study methodology in workplace health promotion programs," American Journal of Health Promotion, vol. 28, no. 6, pp. 347-363, 2014.

[116] P. M. Wayne, D. P. Kiel, J. E. Buring et al., "Impact of Tai Chi exercise on multiple fracture-related risk factors in postmenopausal osteopenic women: a pilot pragmatic, randomized trial," BMC Complementary and Alternative Medicine, vol. 12, article 7, 2012.

[117] P. M. Wayne, B. Manor, V. Novak et al., "A systems biology approach to studying Tai Chi, physiological complexity and healthy aging: design and rationale of a pragmatic randomized controlled trial," Contemporary Clinical Trials, vol. 34, no. 1, pp. 21-34, 2013.

[118] Physician assistant and advance practice nurse care in hospital outpatient departments: United States, 2008-2009 (NCHS data brief), http://www.cdc.gov/nchs/data/databriefs/db77.htm. 


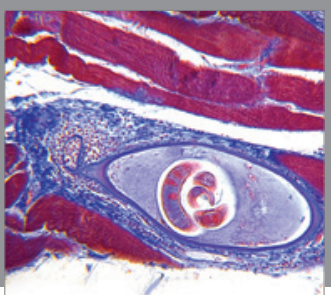

Gastroenterology

Research and Practice
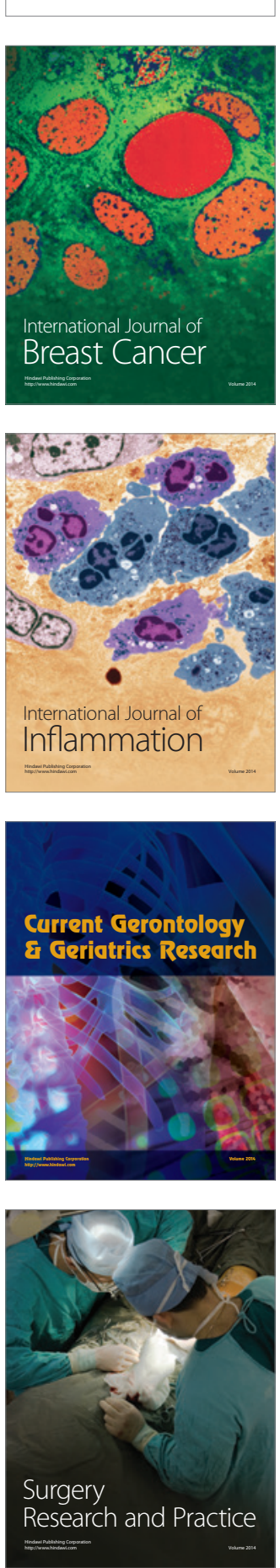

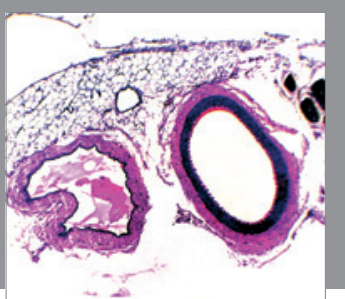

International Journal of Hypertension
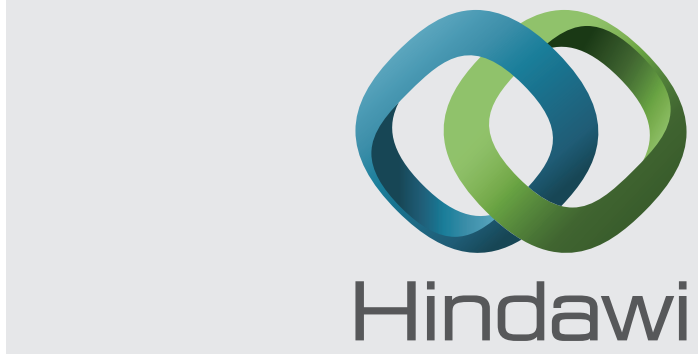

Submit your manuscripts at http://www.hindawi.com
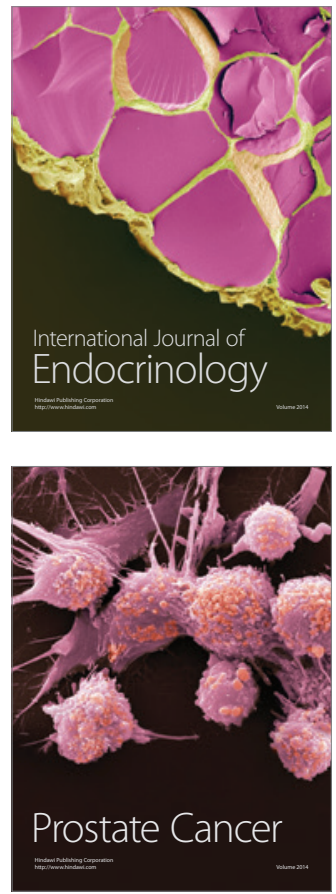

The Scientific World Journal
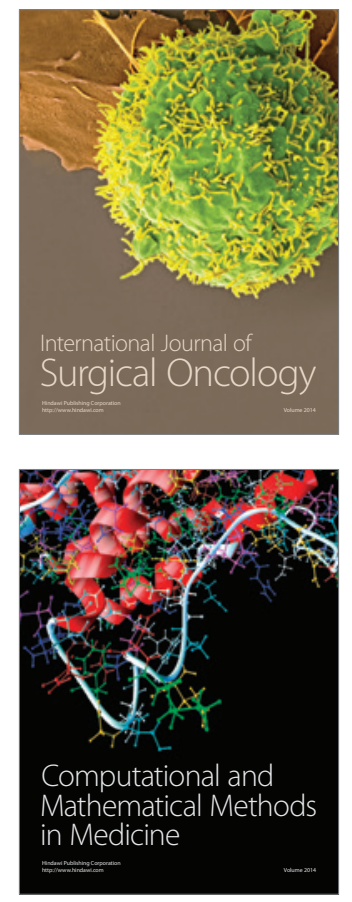
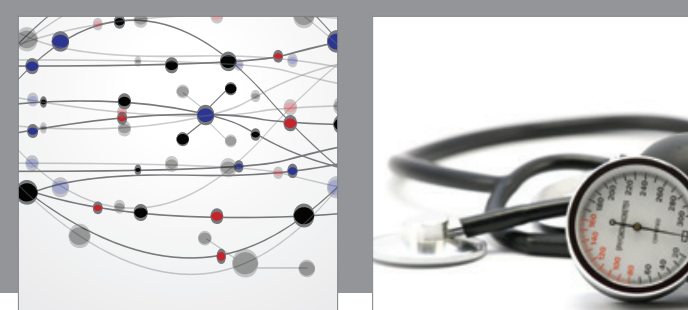

Nursing

Research and Practice

Research and Practice
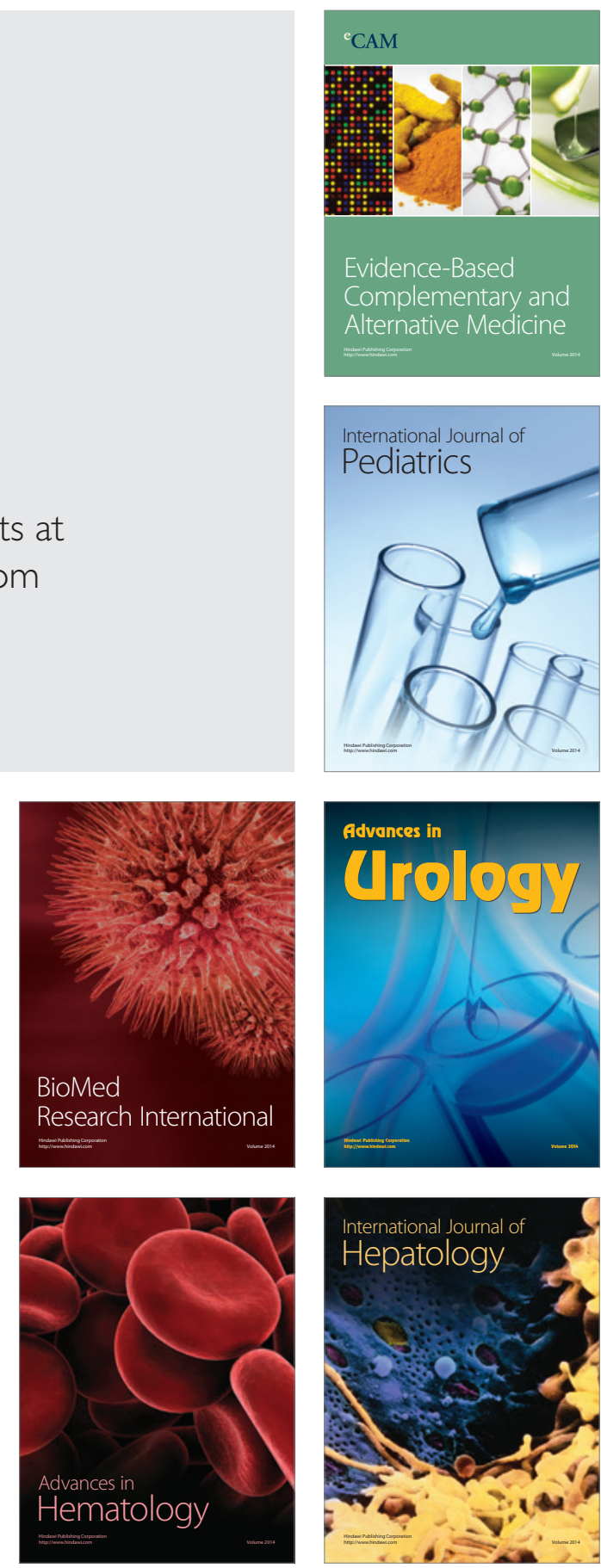
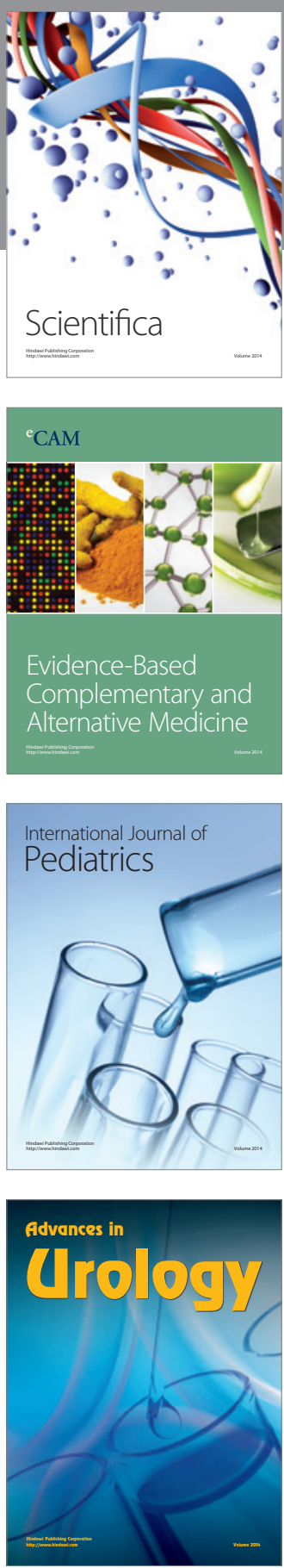

Scientifica

Evidence-Based

Complementary and

Alternative Medicine

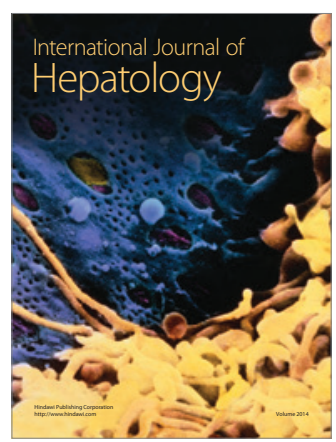

